\title{
Effects of autologous adipose stem cells on dermis layer thickness and fibroblast cells number in photoaging nude mice model.
}

\author{
Li Yu*, Weiwei Yuan, Jingqing Liu
}

Department of Plastic and Cosmetic Surgery, Shenzhen People's Hospital, Second Affiliated Hospital of Jinan University Medical College, Shenzhen, PR China

\begin{abstract}
Objective: To investigate the effects of autologous Adipose Stem Cells (ASCs) on the thickness of dermis and the number of fibroblasts in nude mice. As a result, relevant experimental basis was provided for clinical application of autologous ASCs in skin photoaging therapy.

Methods: 50 SPF grade balb/c nude mice were selected and divided into 5 groups: normal group $(n=10)$, photoaging model group $(n=10)$, photoaging+ASCs group $(n=10)$, photoaging+PBS group $(n=10)$, photoaging+aminoguanidine group $(n=10)$. In addition to the normal group, the remaining groups were constructed by UV irradiation to establish a photoaging model. After successful modeling, the mice in the ASCs group were injected with $1 \mathrm{ml}$ ASCs (at a concentration of $1 \times 10^{6} \mathrm{cells} / \mathrm{ml}$ ); the nude mice in the photoaging+PBS group were performed intradermal injection of equal dose of PBS. The mice in the photoaging+aminoguanidine group were performed intradermal injection of aminoguanidine $(100 \mathrm{mg} /$ $\mathrm{kg}$ ). After $4 \mathrm{w}$ of feeding, dorsal skin in each group was observed. The thickness of the dermis was observed by Masson staining, and the number of fibroblasts was counted.

Result: The skin gloss and elasticity of the nude mice were significantly lower than those in the normal group. The skin gloss and elasticity of photoaging+ASCs group and photoaging+aminoguanidine group were significantly higher than those of the normal group. Compared with the normal group, the dermal thickness and the number of fibroblasts decreased in photoaging group $(P<0.05)$, which accorded with the characteristics of photoaging; the dermal thickness and the number of fibroblasts were significantly increased $(\mathrm{P}<0.05)$ in the photoaging+ASCs group and photoaging+aminoguanidine group. There was no significant difference in the number of dermal thickness and fibroblasts between the PBS group and the model group $(\mathbf{P}>\mathbf{0 . 0 5})$.

Conclusion: ASCs transplantation can significantly increase the dermal layer thickness of nude mice and increase the number of fibroblasts. ASCs transplantation can be used to improve skin aging caused by light injury.
\end{abstract}

Keywords: Autologous adipose stem cells, Photoaging, Dermis, Fibroblasts.

Accepted on November 2, 2017

\section{Introduction}

External environmental factors such as ultraviolet radiation, wind, sun and other skin aging caused by aging are often referred to as exogenous aging, as opposed to intrinsic aging. Ultraviolet radiation in sunlight is the most important factor causing exogenous skin aging, so ultraviolet radiation caused by skin aging can also be called photoaging. Long-term sunlight can lead to changes in the cell composition of the skin, as well as the organizational structure, while the changes in the dermis composition caused by light are most characteristic. Photoaging mainly caused thinning of dermis thickness, decreased fibroblasts number and synthesis function, showing dry skin, increased wrinkles and decreased skin elasticity. Adipose tissue-derived stem cells (ASCs) [1] can secrete a variety of cytokines. At present, a large number of experimental studies have proved that autologous adipose stem cells have a certain anti-aging effect restoring skin elasticity and inhibiting wrinkles, but the specific mechanism is not clear [2]. In this study, by establishing photoaging nude mouse model, the effects of ASCs on the thickness of dermis and the number of fibroblasts in nude mice were observed. The experimental basis for adipose-derived stem cells in anti-aging therapy was provided.

\section{Materials}

\section{Experimental animal source}

Ethical approval was given by the medical ethics committee of Shenzhen People's Hospital, Second Affiliated Hospital of Jinan University Medical College with the following reference number: 2016016.50 Health class SPF class balb/c nude mice 
are weighed 20-22 g with 25 male and 25 female. All animals are raised in our laboratory animal center.

\section{Major reagents and instruments}

TN-2340 UV illuminometer; Optical microscope; Microscope eyepiece micrometer produced in Wuhan; Type I Collagenase: Gibco Corporation.

\section{Experimental grouping}

60 SPF grade balb/c nude mice randomly were divided into normal group (10), photoaging model group (10), photoaging +ASCs group (10), photoaging+PBS group (10), and photoaging+aminoguanidine group (10). Nude mice in normal group were given routine feeding, while the remaining four groups were molded with skin photoaging.

\section{Methods}

Construction of photoaging nude mouse model: Photoaging nude mouse models were built according to the relevant literature and pre-experimental results $[3,4]$. Ultraviolet radiation method: the nude mice in 4 groups were given ultraviolet radiation $180 \mathrm{~m} \mathrm{~J} / \mathrm{cm}^{2}$ in the first week. The energy in the $2-4 \mathrm{w}$ was 240,300 and $360 \mathrm{~m} \mathrm{~J} / \mathrm{cm}^{2}$, respectively. The energy maintained $360 \mathrm{~m} \mathrm{~J} / \mathrm{cm}^{2}$ in 5 to $8 \mathrm{w}$. Daily exposure was $30 \mathrm{~min}$ in the first week, and increased to $60 \mathrm{~min} / \mathrm{d}$ after a week.

Isolation and culture of autologous adipose ASCs: In this study, autologous bilateral inguinal fat of nude mouse was selected as tissue source for separation, culture and passage. The specific procedure was discussed below. Firstly, bilateral groin fat pad of intact nude mouse was placed in a petri dish. It was then repeatedly rinsed with PBS, and $0.1 \%$ type I collagenase was adopted for digestion, shock and centrifugation. The precipitation in the bottom was taken and resuspended, leaving the cell suspension inoculated in the culture dish, and cultured in $5 \% \mathrm{CO}_{2}$ incubator at room temperature. The medium was changed after 24-48 h, and nonadherent cells were removed. When the fusion cells reached $80 \%, 0.05 \%$ trypsin was used for digestion passage. After ASCs passaging to the third generation, trypsin digestion, centrifugation and PBS resuspension were performed, resulting in a solution of concentration at $1 \times 10^{6} / \mathrm{ml}$ for standby application.

Intradermal injection of ASCs: The mice in the photoaging + ASCs group were injected with $1 \mathrm{ml} \mathrm{ASCs}\left(1 \times 10^{6}\right.$ cells $\left./ \mathrm{ml}\right)$ in the dorsal dermis (concentration of $1 \times 10^{6} / \mathrm{ml}$ ); photoaging +PBS group nude mice were given dorsal intradermal injection of equal dose PBS; the nude mice in the photoaging + PBS group were performed dorsal intradermal injection of equal dose of PBS. The mice in the photoaging+aminoguanidine group were performed intradermal injection of aminoguanidine $(100 \mathrm{mg} / \mathrm{kg})$. The mice in the above groups were sacrificed after $4 \mathrm{w}$. The skin tissues were fixed with $4 \%$ paraformaldehyde and embedded in paraffin. The slice thickness was about $5 \mu \mathrm{m}$.

\section{Histological examination}

The nude mice skin sections in all groups were masson stained. The dermis thickness of each group was observed under optical microscope. The number of fibroblasts and the expression of collagen in each skin tissue were recorded. The number of fibroblasts was counted according to the formula: number of cells $/ \mathrm{mm} 2=$ total number of cells $/ \mathrm{n} / \mathrm{N} / 0.005 . \mathrm{n}=$ number of field of view, $\mathrm{N}=$ cases number, $0.005=$ area of formatted eyepiece micrometer.

\section{Statistical processing}

SPSS19.0 software was used for statistical analysis. The results were analysed by one-way ANOVA and $\mathrm{P}<0.05$ was considered statistically significant.

\section{Result}

\section{Animal behavioral performance}

Back skin of nude mice in normal group was moist and elastic with no significant wrinkles. In photoaging group, skin color of nude mice was dim and short of gloss, companied with dry skin with obvious wrinkles. Some nude mice have peeling or damage on the back, in line with the performance of photoaging skin. Compared with the model group, mice in photoaging+ASCs group had decreased skin wrinkles, with moist skin and a certain degree of gloss and elasticity, showing significant improvement. Mice in photoaging+PBS group showed no significant improvement in the depth of skin wrinkles and gloss, and in the degree of moisture. In photoaging+aminoguanidine group and photoaging+ASCs group, the nude mice showed significant improvement in skin color, moisture, glossiness and wrinkle depth than model group (Figure 1).

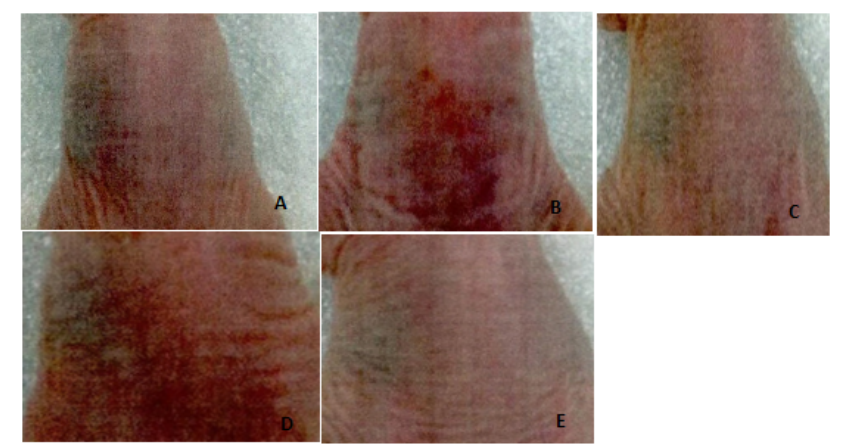

Figure 1. Back skin appearance of nude mice; A: normal group; B: photoaging group model; $C$ : photoaging $+A S C$ sroup; D: phtoaging $+P B S$ group; E: photoaging+aminoguanidine group.

\section{Comparison of dermal skin thickness and fibroblasts numbers of nude mice sample in each group}

Dermal skin thickness of normal group, photoaging group, photoaging+ASCs group, phtoaging+PBS group, photoaging + aminoguanidine group was $(231.2 \pm 2.29) \mu \mathrm{m},(141.2 \pm 3.35$ $\mu \mathrm{m}),(221.2 \pm 4.0 \mu \mathrm{m}),(142.0 \pm 5.24 \mu \mathrm{m})$ and $(210.0 \pm 3.14$ 
$\mu \mathrm{m})$, respectively. Compared with the normal group, the thickness of dermis decreased significantly, showing statistically significant difference $(\mathrm{P}<0.05)$, which accorded with the characteristic of photoaging. Dermal thickness of photoaging+ASCs group and photoaging+aminoguanidine group increased significantly with statistical significant difference $(\mathrm{P}<0.05)$. There was no significant difference in dermal thickness between the photoaging+PBS and the model group $(\mathrm{P}>0.05)$. In addition, there was no significant difference in the thickness of dermis between the photoaging+ASCs group and the photoaging+aminoguanidine group $(\mathrm{P}>0.05)$ (Table 1 and Figure 2).
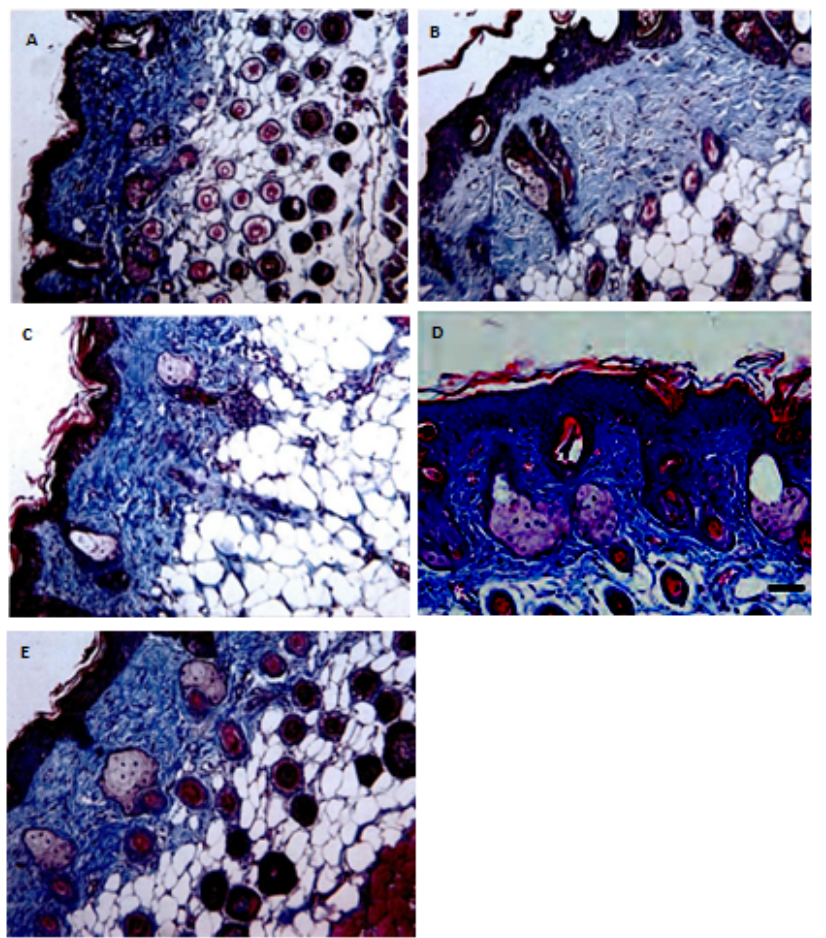

Figure 2. Histopathological changes of nude mice skin (Masson staining X100); A: normal group; B: photoaging model group; $C$ : photoaging $+A S C$ sroup; D: photoaging $+P B S$ group; E: photoaing +aminoguanidine group.

Under light microscopic, wavy collagen fibers could be seen in dermis layer of nude mice, with dark stain, homogenous distribution and a large number of fibroblasts. The nude mice in photoaging group had uneven stained skin collagen fibers, with inhomogeneous distribution and irregular rearrangement. Besides, the number of fibroblasts in nude mice was significantly lower than that in normal tissues $(\mathrm{P}<0.05)$. Skin of nude mice in photoaging+ASCs group had deeper stained collagen fibers than those in normal group. The fibers were wavy and closely distributed with regular form, containing more fibroblasts than model group $(\mathrm{P}<0.05)$. Skin of nude mice in photoaging+PBSs group had similar collagen fibers with model group. The fibers had irregular form, some even in bulk, and little fibroblasts, showing no significant difference between the two groups $(\mathrm{P}>0.05)$. Skin of nude mice in photoaging+aminoguanidine group had homogenous stained collagen fibers that were arranged closely. The result was similar with that in photoaging+ASCs group, showing no significant difference $(\mathrm{P}>0.05)$ (Table 1 and Figure 2$)$.

Table 1. Comparison of dermal thickness and number of fibroblasts in dorsal skin of nude mice in each group.

\begin{tabular}{lllll}
\hline Groups & Cases & $\begin{array}{l}\text { Dermal } \\
(\mu \mathrm{m})\end{array}$ & thickness & $\begin{array}{l}\text { Number } \\
\text { fibroblasts }\end{array}$ \\
\hline Normal & 10 & $231.2 \pm 2.29^{*}$ & $\begin{array}{l}911.55 \\
132.96^{*}\end{array}$ & \pm \\
\hline Photoaging & 10 & $141.2 \pm 3.35$ & $672.71 \pm 67.22$ \\
\hline Photoaging+ascs & 10 & $221.2 \pm 4.0^{*}$ & 899.98 & \pm \\
\hline Photoaging+pbs & 10 & $142.0 \pm 5.24^{\#}$ & $677.62 \pm 70.11^{\#}$ \\
\hline $\begin{array}{l}\text { Photoaging } \\
\text { +aminoguanidine }\end{array}$ & 10 & $210.0 \pm 3.14^{*}$ & 881.69 & \pm \\
\hline
\end{tabular}

\section{Discussions}

UVA and UVB are the most important spectrums in ultraviolet light causing skin light damage and aging. Moreover, UVB can cause DNA damage, skin aging or damage, and even induce skin cancer. In this way, the prevention and treatment of skin damage caused by light has now become a research hotspot.

ASCs [5,6] are source of mesenchymal stem cells in mesoblast, which have multi-directional differentiation potential and play an important role in the field of tissue engineering seed cell research. It is generally believed that autologous fat is the best tissue filling material, and a large number of studies $[7,8]$ have found that ASCs have a high effectiveness in anti-aging, soft tissue injury repair, and promoting grafted fat survival. Some scholars have found that subcutaneous injection of adipose tissue can improve skin aging $[9,10]$, thus producing new collagen and keeping the skin firm. In recent years, it has been clarified that ASCs could activate fibroblasts by secreted cytokines for skin aging treatment. Through differentiation, type I, IV and V collagens were stimulated, and fibroblasts were reorganized to increase the matrix composition of skin cell, so as to achieve the effect of dermal tissue and skin structure repair $[11,12]$. In this study, we observed the effect of autologous ASCs on the thickness of dermis and the number of fibroblasts by constructing nude mice photoaging group.

In this study, the photoaging model was constructed by ultraviolet irradiating the dorsal skin of nude mice. Compared with normal nude mice, skin color of photoaging nude mice was pale and short of luster. Besides, some nude mice had obvious wrinkles and even mild erythema at the back. Tissue staining showed the dermal layer of photoaging nude mice was thinning with dermal cells arranged irregularly. Collagen fibers were uneven stained, with inhomogeneous distribution and irregular rearrangement. The number of fibroblasts was significantly reduced in dermis. After injection of ASCs in the dermis, the gloss and skin color were improved. Histopathological manifestations of the dermis layer thickness were similar to that in normal group with no significant difference. It was found that collagen fibers were rich in dermis 
layer and were neatly arranged after injection. The number of fibroblasts was significantly higher than that of the model group, and there's no significant difference with normal group.

Advanced glycosylation of the final product by nonenzymatic glycosylation made the protein brown, fluorescent and crosslinked, promoting all kinds of aging symptoms. It was found that non-enzymatic glycosylation on the skin's aging mainly relied on collagen fibers and elastic fibers, resulting in wrinkles and decreased skin elasticity. Aminoguanidine [13,14] is an inhibitor of advanced glycosylation final products, which has been found to promote angiogenesis and wound healing in previous studies and is currently an important research spot in delaying skin aging. Therefore, in this study, aminoguanidine was selected as a positive control group. The study also found that in photoaging+aminoguanidine group and photoaging +ASCs groups, dermal layer thickness and fibroblast cells numbers were significantly higher than those in the model group. The indications were similar to those in normal group. The above results showed that ASCs transplantation could significantly increase the thickness of dermal layer in nude mice, as well as the number of fibroblasts. In a word, ASCs transplantation can be used to prevent skin aging.

A variety of factors were considered for clinical use of stem cells, such as how to differentiate stem cells with differentiation ability and establish an effective threedimensional structure to ensure the survival and tissue regeneration of differentiated cells $[15,16]$. Although ASCs have been shown to have good anti-aging effects in the experiment, their safety, stability and mechanisms related to anti-photoaging have not been fully elucidated, which will be the focus of future research.

\section{References}

1. Hur W, Lee HY, Min HS. Regeneration of full-thickness skin defects by differentiated adipose-derived stem cells into fibroblast-like cells by fibroblast-conditioned medium. Stem Cell Res Ther 2017; 8: 92

2. Wang Y, Chu Y, Yue B. Adipose-derived mesenchymal stem cells promote osteosarcoma proliferation and metastasis by activating the STAT3 pathway. Oncotarget 2017; 8: 23803-23816.

3. Zhang SC. Study of adipose-derived stem cells antagonizing D-galactose-induced skin senescence. South Med Univ 2014.

4. Chen XL. Study on the effect of herbal medicine dried tangerine on skin aging. Guangzhou Univ Trad Chinese Med 2015.

5. Xu X. Effects of adipose-derived stem cell transplantation on skin photoaging and its mechanism. Second Milit Med Univ 2014.

6. Yao Y, Dong Z, Liao Y. Adipose extracellular matrix/ stromal vascular fraction gel: a novel adipose tissue-derived injectable for stem cell therapy. Plast Reconstr Surg 2017; 139: 867-879.

7. Zhou Q, Li B, Zhao J. IGF-I induces adipose derived mesenchymal cell chondrogenic differentiation in vitro and enhances chondrogenesis in vivo. In Vitro Cell Dev Biol Anim 2016; 52: 356-364.

8. Kevin D, Taegyn T, Andrew W. Fibroblast-derived extracellular matrix induces chondrogenic differentiation in human adipose-derived mesenchymal stromal/stem cells in vitro. Int J Mol Sci 2016; 17.

9. Huang HZ, Li W, Xu P. Experimental study on improving the skin texture of nude mice with nano-fat. J Tissue Eng Reconstr Surg 2016; 4: 212-216.

10. Jin $M$, Shen X, Zhao C. In vivo study of effects of artesunate nanoliposomes on human hepatocellular carcinoma xenografts in nude mice. Drug Deliv 2013; 20: 127.

11. Minjuan W, Jun X, Shiyun S. Hair follicle morphogenesis in the treatment of mouse full-thickness skin defects using composite human acellular amniotic membrane and adipose derived mesenchymal stem cells. Stem Cells Int 2016; 2016: 8281235 .

12. Dizaji Asl K, Shafaei H, Soleimani Rad J. Comparison of characteristics of human amniotic membrane and human adipose tissue derived mesenchymal stem cells. World J Plast Surg 2017; 6: 33-39.

13. Peng LW, Lai JX, He CF. Advances in nonenzymatic glycosylation and skin aging. Chinese J Gerontol 2010; 20: 3027-3029.

14. Patrick JS, Thorpe SR, Baynes JW. Nonenzymatic glycosylation of protein does not increase with age in normal human lenses. J Gerontol 1990; 45: 18.

15. Ge K, Lu SL, Qing C. Study on the effect of aminoguanidine on the wound healing of diabetic patients. Chinese J Traumatol 2004; 8: 17-21.

16. Bliley JM, Argenta A, Satish L. Administration of adiposederived stem cells enhances vascularity, induces collagen deposition, and dermal adipogenesis in burn wounds. Burns 2016; 42: 1212-1222.

\section{${ }^{*}$ Correspondence to}

$\mathrm{Li} \mathrm{Yu}$

Department of Plastic and Cosmetic Surgery

Shenzhen People's Hospital

Second Affiliated Hospital of Jinan University Medical College

PR China 